\title{
Association between serum cystatin $C$ and diabetic peripheral neuropathy: a cross-sectional study of a Chinese type 2 diabetic population
}

\author{
Yanyun Hu${ }^{1,2}$, Fang Liu ${ }^{1,2}$, Jing Shen ${ }^{1,2}$, Hui Zeng ${ }^{1}$, Lianxi $\mathrm{Li}^{1,2}$, Jun Zhao ${ }^{3}$, \\ Jungong Zhao ${ }^{4}$, Fengdi Lu' ${ }^{1}$ and Weiping Jia ${ }^{1,2}$ \\ ${ }^{1}$ Department of Endocrinology and Metabolism, Shanghai Jiaotong University Affiliated Sixth \\ People's Hospital, Shanghai, China, ${ }^{2}$ Shanghai Key Laboratory of Diabetes, Shanghai Institute of Diabetes, \\ Shanghai Clinical Medical Center of Diabetes, Shanghai Key Clinical Center of Metabolic Diseases, Shanghai, China, \\ ${ }^{3}$ Departments of Vascular Surgery and ${ }^{4}$ Interventional Radiology, Shanghai Jiaotong University Affiliated Sixth \\ People's Hospital, Shanghai, China
}

\begin{abstract}
Objective: Serum cystatin $\mathrm{C}$ (CysC) is a sensitive marker of kidney function and recent studies have shown that Cys $\mathrm{C}$ plays a critical role in degenerative diseases in both the central and the peripheral nervous systems. The aim of this study was to explore the relationship between serum CysC and diabetic peripheral neuropathy (DPN) in patients with type 2 diabetes. Methods: In total, 937 type 2 diabetic patients were enrolled in this cross-sectional study. Serum CysC concentration was measured by immunoturbidimetry. DPN was evaluated by neurological symptoms, neurological signs, neurothesiometer, and electromyogram.
\end{abstract}

Results: Serum CysC levels were significantly higher in DPN patients (1.3 (1.1-1.5) $\mathrm{mg} / \mathrm{l})$ compared with patients with signs of DPN (1.1 (0.9-1.3) mg/l, $P<0.001)$ and non-DPN patients $(1.0(0.9-1.3) \mathrm{mg} / \mathrm{l}, P<0.001)$. Multiple regression analysis revealed that DPN was associated with age, diabetes duration, $\mathrm{HbA1C}$, and serum CysC. Spearman's correlation analysis showed that serum CysC was closely related with age, sex, diabetes duration, hypertension, glomerular infiltration rate, and serum creatinine $(\mathrm{Cr})$ level. The patients were divided into quartiles according to the serum CysC levels. Compared with quartile 1 (referent), the risk of DPN was significantly higher in quartile 2 (odds ratio (OR), 1.753; 95\% Cl, 1.055-2.912; $P<0.05$ ), quartile 3 (OR, 2.463; 95\% Cl, 1.445-4.917; $P<0.01$ ), and quartile 4 (OR, 5.867; 95\% Cl, 2.075-16.589; $P<0.01$ ). Receiver-operating characteristic analysis revealed that the optimal cutoff point of serum CysC to indicate DPN was $1.25 \mathrm{mg} / \mathrm{ln}$ male patients and $1.05 \mathrm{mg} / \mathrm{l}$ in female patients. High serum CysC level indicated a onefold higher risk of DPN.

Conclusions: High serum CysC level is closely associated with DPN and may be a potential biomarker for DPN in type 2 diabetic patients.

European Journal of

Endocrinology

(2014) 171, 641-648

\section{Introduction}

Diabetic peripheral neuropathy (DPN) is one of the most common complications of diabetes mellitus. And DPNassociated diabetic foot is a leading cause of non-traumatic amputation, which results in diminished life quality of diabetic patients. Previous studies revealed that DPN affected up to $50 \%$ of diabetic patients (1). In view of the large diabetes population and sharp rise in morbidity of diabetes worldwide, DPN has posed a great challenge for medical professionals and society. Although the pathogenesis of DPN still has not been elucidated, several risk factors including hyperglycemia, dyslipidemia, smoking, etc. have been identified (2). Moreover, vascular diseases including cardiovascular disease, peripheral vascular disease, diabetic nephropathy, and diabetic retinopathy were all found to be risk factors of DPN $(2,3)$. A population-based study of type 2 diabetic patients in Sweden showed that the prevalence of peripheral sensory

Published by Bioscientifica Ltd 
neuropathy increased with the severity of retinopathy and overt nephropathy, indicating that peripheral sensory neuropathy was associated with micro- or macro-angiopathy in type 2 diabetes (4). It has also been proved that mircroalbuminuria, an indicator of microvascular complications especially for diabetic patients, was closely related to DPN (5).

Cystatin C (CysC) is a small molecular protein that is produced by all nucleated cells at a constant rate, freely filtered in the glomeruli and almost completely reabsorbed in the distal tubule. It has been suggested that serum CysC levels were generally not affected by extra-renal factors such as age, sex, and muscle mass (6). Therefore, serum CysC is now considered as an alternative or even better estimator of glomerular filtration rate (GFR) especially in patients with normal or mild-to-moderate renal dysfunction (7). Recent studies have found that CysC was a stronger predictor of the risk of death and cardiovascular events in elderly persons (8). In patients with coronary heart disease, high CysC concentrations predicted substantial increased risks of allcause mortality, cardiovascular events, and incident heart failure (9). Some studies also proved that CysC played an important role in the diseases of both central and peripheral nervous diseases $(10,11)$. In the central nervous systems (CNS), CysC was mainly located in neurons and microglia, functioning as a cysteine protease inhibitor, and can be released to extra-cellular space upon injury. It has been proved that $\mathrm{Cys} C$ regulated the aggregation and deposition of amyloid $\beta$ (12). And the serum CysC levels in patients with Alzheimer's disease were found be significantly higher than healthy controls (13). Moreover, change in the concentration of CysC in cerebrospinal fluid was also observed in demyelinating diseases including multiple sclerosis and Guillain Barre syndrome. The alteration in cerebrospinal fluid CysC concentration in these diseases was considered a biomarker of nerve injury, and serum CysC was mainly released by affected neurons and microglia to clear debris and improve the following nerve regeneration $(11,14)$. In addition, our previous studies have demonstrated that serum CysC was a strong marker for lower limb ischemia and diabetic retinopathy in Chinese type 2 diabetic patients $(15,16)$. Therefore, we carried out this study to investigate the association between CysC and DPN in type 2 diabetic patients.

\section{Materials and methods}

\section{Study population}

In this study, we enrolled consecutive 937 diabetic inpatients at the Shanghai Clinical Medical Center of
Diabetes from January 2012 to December 2012. They were mainly local from 16 districts of Shanghai and were admitted for uncontrolled hyperglycemia and diabetic complications. The diagnostic criteria of type 2 diabetes mellitus was based on American Diabetes Association standards (17). GFR $<60 \mathrm{ml} / \mathrm{min}$ per $1.73 \mathrm{~m}^{2}$ was considered renal dysfunction. For 3 consecutive days, 24-h urine samples were collected for the assessment of 24-h urinary albumin levels. And at least two of three samples with urinary albumin $\geq 30 \mathrm{mg} / 24 \mathrm{~h}$ were defined as persistent urinary albuminuria. The patients with the following conditions were excluded: i) acute complications of diabetes including diabetic hyperosmolar coma, ketoacidosis, and acute foot ulcer; ii) history of cerebral infarction; and iii) complicated with degenerative changes in cervical vertebra.

DPN was categorized as follow: i) DPN, patients had both clinically evident DPN (defined as at least two positive findings among sensory symptoms, signs, or reflex abnormalities consistent with a distal symmetrical polyneuropathy) and abnormal results on nerve conduction tests (defined by the presence of at least one abnormal nerve attribute (of amplitude, latency, F-wave, or nerve conduction velocity) in two or more nerves among the median, peroneal, and sural nerves); ii) signs of DPN, patients had either clinical evident DPN or abnormal results on nerve conduction tests; and iii) non-DPN, patients had neither clinical evident DPN nor abnormal nerve conduction tests (18).

All the enrolled patients continued their previous glycemic control regimen including hypoglycemic drugs and(or) insulin except in the very morning when their overnight fasting and $2-\mathrm{h}$ postprandial blood samples were collected. They also continued to use anti-hypertensives and lipid-regulating agents if necessary. The study was approved by the Ethics Committee of Shanghai Clinical Medical Center of diabetes. Written informed consents were obtained from all participants and adhered to the tenets of the Declaration of Helsinki.

\section{Data collection}

Information of smoking behavior, alcohol consumption, and hypertension was obtained by a standardized questionnaire. Height and weight were assessed on a standardized form by the same physician during the health check-up. BMI was calculated as body weight (kg) divided by the square of the height (m). All the patients had an overnight fast before blood and urine samples were collected. The samples were stored at $4{ }^{\circ} \mathrm{C}$ if the assays 
could not be performed immediately. All samples were analyzed for biochemical characteristics including CysC within $48 \mathrm{~h}$ after collection in the medical examination center of Shanghai Sixth People's Hospital and the laboratory of Shanghai Clinical Medical Center of Diabetes.

\section{Laboratory measurements}

Blood samples were transported to the medical examination center of Shanghai Sixth People's Hospital or the laboratory of Shanghai Clinical Medical Center of Diabetes as needed after collected. HbA1c level was determined by high-pressure liquid chromatography and glycated albumin (GA) was measured by the liquid enzymatic assay. Concentrations of serum creatinine $(\mathrm{Cr})$ and serum lipids including total cholesterol (TC), triglyceride (TG), HDL cholesterol (HDL-cholesterol), LDL cholesterol (LDL-cholesterol) were analyzed by enzymatic method. Serum CysC concentration was determined by high-sensitive latex-enhanced immune-turbidimetric method (19) using an automatic biochemical analyzer (7600-020; Hitachi, Inc.). Urinary albumin was measured by RIA. The GFR was determined by technetium- $99 \mathrm{~m}$ diethyl triamine penta-acetic acid clearance.

\section{Neuropathy assessment}

An evaluation of neuropathy was performed in the patients, including recognizing the neurological symptom signs, examining the ankle and knee reflex, measuring the vibration perception threshold (VPT) value, and conducting the electromyogram. Twenty-four hours before and during the neurological examination, the confounding influencing factors (including spicy food, caffeinecontaining drinks, physical activities, emotional stress, and insomnia) were avoided. All the tests were carried out in a quiet special room by the same physician or technician.

Neurological symptoms and signs $>$ A complete history of neurological symptoms of each patient was taken at the visit. The assessment of neurological symptoms and signs was based on the Toronto Clinical Scoring System (20). Any pain, numbness, tingling, weakness of foot, or ataxia or upper-limb symptoms were considered positive symptoms. The knee and ankle reflex examinations were also performed by the same physician.

VPT assessment $\downarrow$ In each patient, the VPT values were measured by a neurothesiometer (Bio-Thesiometer; BioMedical Instrument Co., Newbury, OH, USA). The operational approaches were based on the International Working Group on the Diabetic Foot of the International Diabetes Federation (21) and in accordance with our previous study (22). VPT value higher than $25 \mathrm{~V}$ (volt) on either limb was considered abnormal.

Nerve conduction velocity tests $\bullet$ Electromyogram (Myto, EBNeuro, Firenze, Italy) was performed to assess the median, ulnar, tibial, common peroneal, and superficial peroneal nerve conduction velocity (NCVs) on both sides of each subject. The patients stayed calm and relaxed, and local skin temperature was kept constant $\left(32-33^{\circ} \mathrm{C}\right)$ during the tests. Briefly, the motor NCVs of median, ulnar, tibial, and common peroneal nerves and the sensory NCV of median, ulnar, common peroneal, and sural nerves were determined. The threshold for decreased NCVs was set according to the NCVs reference value of Chinese people (23). The latency of each nerve, F-wave, and $\mathrm{H}$ reflex was also recorded.

\section{Statistical analysis}

For continuous variables, results were presented as mean \pm S.D. or median (25th-75th percentiles), and differences between groups were evaluated by Student's $t$-test or MannWhitney $U$ test. Categorical variables were presented as frequency percentage, and intergroup comparisons were analyzed using a $\chi^{2}$-test. Spearman's correlation analysis was also performed to explore the interrelationship between different clinical characteristics. Multiple logistic regression analysis was used to evaluate the relationship between DPN and different clinical characteristics. Multiple regression analysis was used to investigate the association between DPN and clinical variables. The patients were also categorized into quartiles based on the serum CysC level: quartile 1 , CysC $<$ $0.9 \mathrm{mg} / \mathrm{l}$; quartile $2,0.9 \mathrm{mg} / \mathrm{l} \leq \mathrm{CysC} \leq 1.1 \mathrm{mg} / \mathrm{l}$; quartile 3 , $1.2 \mathrm{mg} / \mathrm{l} \leq$ CysC $\leq 1.3 \mathrm{mg} / \mathrm{l}$; quartile 4 , CysC $>1.3 \mathrm{mg} / \mathrm{l}$. Logistic regression analysis was used to evaluate the risk of DPN in different CysC quartiles. Furthermore, receiveroperating characteristic (ROC) analysis was performed to identify the optimal cutoff point of CysC for indicating DPN. The risk of DPN in patients with high CysC levels, albuminuria, and renal dysfunction was also evaluated using

Table 1 Main diagnosis for admission of patients.

\begin{tabular}{lcc}
\hline Main diagnosis & Case $n(\%)$ \\
\hline Uncontrolled hyperglycemia & & $578(61.70)$ \\
Diabetic peripheral neuropathy & $246(26.25)$ \\
Diabetic nephropathy & $66(7.04)$ \\
Diabetic foot & $47(5.02)$ \\
\hline
\end{tabular}


Table 2 Characteristics of patients with non-DPN, signs of DPN, and DPN.

\begin{tabular}{l}
\hline \\
\hline Age (years) \\
Sex male (\%) \\
Diabetes duration (years) \\
Smoking (\%) \\
Drinking (\%) \\
Hypertension (\%) \\
BMI $\left(\mathrm{kg} / \mathrm{cm}^{2}\right)$ \\
TC $(\mathrm{mmol} / \mathrm{l})$ \\
TG $(\mathrm{mmol} / \mathrm{l})$ \\
HDL-cholesterol (mmol/l) \\
LDL-cholesterol (mmol/l) \\
HbA1c (\%) \\
GA (\%) \\
Cystatin C (mg/l) \\
Cr $(\mu \mathrm{mol} / \mathrm{l})$ \\
GFR (ml/min/1.73 $\left.\mathrm{m}^{2}\right)$ \\
Urinary albumin $(\mathrm{mg} / 24 \mathrm{~h})$
\end{tabular}

\begin{tabular}{c}
\hline Non-DPN $(n=351)$ \\
\hline $57.46 \pm 11.33$ \\
56.7 \\
$8.00(4.00-13.00)$ \\
32.6 \\
19.1 \\
53.3 \\
$25.35 \pm 3.55$ \\
$4.63(3.95-5.31)$ \\
$1.46(1.03-2.10)$ \\
$0.99(0.86-1.19)$ \\
$2.76(2.20-3.25)$ \\
$8.11 \pm 1.88$ \\
$19.80(17.00-24.50)$ \\
$1.0(0.9-1.3)$ \\
$63(53-78)$ \\
$98.99 \pm 24.85$ \\
$64.40 \pm 1.71$
\end{tabular}

\begin{tabular}{c}
\hline Signs of DPN $(n=482)$ \\
\hline $59.49 \pm 12.00$ \\
59.1 \\
$10(4.00-13.00)$ \\
33.3 \\
21.5 \\
55.9 \\
$25.24 \pm 3.48$ \\
$4.64(3.95-5.32)$ \\
$1.44(0.94-2.23)$ \\
$1.03(0.88-1.20)$ \\
$2.70(2.09-3.31)$ \\
$8.56 \pm 1.98$ \\
$21.00(17.70-25.50)$ \\
$1.1(0.9-1.3)$ \\
$67(55-78)$ \\
$93.98 \pm 26.51$ \\
$105.05 \pm 7.68$
\end{tabular}

\begin{tabular}{c}
\hline DPN $(n=104)$ \\
\hline $66.43 \pm 9.67$ \\
54.8 \\
$12.00(7.00-17.75)$ \\
26.9 \\
18.3 \\
75 \\
$25.29 \pm 0.82$ \\
$4.62(3.73-5.28)$ \\
$1.36(0.86-1.87)$ \\
$1.04(0.87-1.28)$ \\
$2.59(2.01-3.19)$ \\
$8.58 \pm 2.17$ \\
$20.60(18.10-25.95)$ \\
$1.3(1.1-1.5)$ \\
$69(59-81)$ \\
$83.51 \pm 22.08$ \\
$226.32 \pm 10.29$ \\
\end{tabular}

\begin{tabular}{c}
\hline $\boldsymbol{P}$ \\
\hline 0.000 \\
NS \\
0.000 \\
NS \\
NS \\
0.000 \\
NS \\
NS \\
NS \\
NS \\
NS \\
0.002 \\
0.029 \\
0.000 \\
0.035 \\
0.000 \\
0.000 \\
\hline
\end{tabular}

TC, total cholesterol; TG, triglyceride; GA, glycated albumin; Cr, creatinine; GFR, glomerular filtration rate; NS: no significance.

logistic regression analysis. All the statistical analyses were performed by SPSS 20.0 (SPSS, Inc.). A two-sided $P<0.05$ was considered statistically significant.

\section{Results}

The main diagnosis of enrolled patients for admission to the Shanghai Clinical Medical Center of Diabetes included uncontrolled hyperglycemia (61.7\%), DPN (26.25\%), diabetic nephropathy (7.04\%), and diabetic foot $(5.02 \%)$ (Table 1). A total of 937 patients (mean age, $59.60 \pm 0.38$ years; male/female, 541/396; and mean diabetes duration, $9.78 \pm 0.23$ years) were finally enrolled in this study. The clinical characteristics of patients with non-DPN, signs of DPN, and DPN are given in Table 2. Compared with patients with signs of DPN and non-DPN, DPN patients were significantly older and had longer diabetes duration (both $P<0.001$ ). The prevalence of hypertension in DPN patients was the highest among three groups $(P<0.001)$. HbA1c $(P=0.002)$, GA $(P=0.029)$, CysC $(P<0.001)$, Cr $(P<0.05)$, and 24-h urinary albumin $(P<0.001)$ levels were all higher, while GFR $(P<0.001)$ was lower in signs of DPN and DPN patients. No significant differences were observed in sex, percentage of smokers, drinkers and BMI, and in TC, TG, HDL cholesterol (HDL-C), and LDL cholesterol (LDL-C) levels. In addition, the mean serum CysC concentration of total number of patients, male patients, and female patients was $(1.20 \pm 0.02) \mathrm{mg} / \mathrm{l},(1.22 \pm 0.02) \mathrm{mg} / \mathrm{l}$, and $(1.16 \pm 0.02) \mathrm{mg} / \mathrm{l}$ respectively. Spearman's correlation analysis showed that serum CysC level was closely associated with age $(r=0.501)$, diabetes duration $(r=$ $0.219)$, hypertension ( $r=-0.247), \mathrm{Cr}(r=0.649)$, and GFR $(r=-0.661)$ (Table 3$)$. Moreover, multiple logistic regression analysis showed that DPN was independently

Table 3 Spearman's correlation analysis of different clinical characteristics.

\begin{tabular}{|c|c|c|c|c|c|c|c|c|c|}
\hline & GFR & $\begin{array}{l}\text { Urinary } \\
\text { albumin }\end{array}$ & $\mathbf{C r}$ & CysC & GAd & HbA1c & Hypertension & $\begin{array}{l}\text { Diabetes } \\
\text { duration }\end{array}$ & Sex \\
\hline Age & $-0.575^{\ddagger}$ & 0.066 & $0.187^{\ddagger}$ & $0.501^{\ddagger}$ & $0.090^{\dagger}$ & 0.025 & $-0.284^{\ddagger}$ & $0.370^{\ddagger}$ & $0.117^{\ddagger}$ \\
\hline Sex & $-0.087^{\dagger}$ & $0.103^{\ddagger}$ & $-0.127^{\ddagger}$ & $-0.124^{\ddagger}$ & 0.009 & 0.036 & -0.064 & $0.085^{\dagger}$ & \\
\hline Diabetes duration & $-0.278^{\ddagger}$ & $0.148^{\ddagger}$ & 0.045 & $0.219^{\ddagger}$ & 0.061 & 0.034 & -0.159 & - & \\
\hline Hypertension & $-0.220^{\ddagger}$ & $-0.243^{\ddagger}$ & $-0.152^{\ddagger}$ & $-0.247^{\ddagger}$ & $0.087^{\dagger}$ & $0.082^{\dagger}$ & - & - & \\
\hline HbA1c & 0.000 & $-0.175^{\ddagger}$ & $-0.136^{\ddagger}$ & $-0.068 *$ & $0.817^{\ddagger}$ & - & - & - & \\
\hline GA & -0.034 & 0.063 & $-0.101^{\dagger}$ & $-0.093^{\dagger}$ & - & - & - & - & \\
\hline CysC & $-0.661^{\ddagger}$ & $0.147^{\ddagger}$ & $0.649^{\ddagger}$ & - & - & - & - & - & \\
\hline $\mathrm{Cr}$ & $-0.444^{\ddagger}$ & -0.048 & - & - & - & - & - & - & \\
\hline Urinary albumin & $-0.138^{\dagger}$ & - & - & - & - & - & - & - & \\
\hline
\end{tabular}


Table 4 Multiple regression analysis of DPN with different clinical characteristics.

\begin{tabular}{|c|c|c|}
\hline Characteristics & Wald & $P$ value \\
\hline Age & 3.895 & 0.048 \\
\hline Diabetes duration & 8.969 & 0.003 \\
\hline Hypertension & 0.660 & NS \\
\hline HbA1c & 5.818 & 0.016 \\
\hline GA & 0.173 & NS \\
\hline CysC & 4.395 & 0.038 \\
\hline $\mathrm{Cr}$ & 0.518 & NS \\
\hline Urinary albumin & 0.144 & NS \\
\hline GFR & 0.055 & NS \\
\hline
\end{tabular}

associated with age $(P<0.05)$, diabetes duration $(P<0.01)$, HbA1c $(P<0.05)$, and CysC $(P<0.05)$. CysC was still associated with DPN after adjustment for age, diabetes duration, HbA1c, Cr, urinary albumin, and GFR $(P<0.05)$ (Table 4). Moreover, Spearman's correlation analysis showed that compared with CysC quartile 1 (referent), patients in quartile 2 (OR, 1.753; 95\% CI, 1.055-2.912; $P<0.05$ ), quartile 3 (OR, 2.463; 95\% CI, 1.445-4.917; $P<0.01)$, and quartile 4 (OR, 5.867; 95\% CI, 2.075$16.589 ; P<0.01$ ) had higher risk of DPN. The risk of DPN, especially in male patients, increased sharply as the serum CysC level elevated (Table 5). Furthermore, ROC analysis revealed that the optimal cutoff point of CysC was $1.25 \mathrm{mg} / \mathrm{l}$ to indicate confirmed DPN (AUC=0.704; 95\% CI, 0.645-0.763; Youden index $=0.305$; sensitivity, 61.8\%; specificity, 68.6\%) in male patients (Fig. 1A) and $1.05 \mathrm{mg} / \mathrm{l}$ to indicate confirmed DPN (AUC $=0.639 ; 95 \%$ CI, 0.559-0.719; Youden index $=0.256$; sensitivity, 70.2\%; specificity, 50.43\%) in female patients (Fig. 1B). Finally, odds ratio (OR) analysis showed that patients with high serum CysC levels (defined as serum Cys C $>1.25 \mathrm{mg} / \mathrm{l}$ in male patients and serum CysC $>1.05 \mathrm{mg} / 1$ in female patients) (male: OR, 2.586; 95\% CI, 1.480-4.519; $P<0.0001$; female: OR, 2.256; 95\% CI, 1.211-4.204; $P<0.0001)$, male patients with albuminuria (OR, 2.26; 95\% CI, (1.10-4.65); $P<0.05)$ and female patients with renal dysfunction (OR, 1.03; 95\% CI, 1.01-1.04; $P<0.01$ ) were all at higher risk of DPN (Table 6).

\section{Discussion}

In general, our study revealed a close relationship between serum CysC levels and DPN in patients with type 2 diabetes. Serum CysC levels of DPN patients were much higher than non-DPN patients, and the elevated serum CysC level was associated with DPN independent of co-variables. More importantly, high serum CysC level indicated increased risk of DPN. Patients with high serum CysC level indicated a onefold higher risk of DPN.

As mentioned earlier, CysC was proved to be related to both CNS and peripheral nervous system diseases in several observational studies (13). Consistent with that, we found a significant increase in serum CysC levels in type 2 diabetic DPN patients. Peripheral nerve fibers are extension of peripheral neurons, which are bundled into groups and surrounded by endoneurium. Just like the bloodbrain barrier, endoneurium separated endoneurial fluid and blood circulation. In diabetic stroke patients, pericyte loss induced by hyperglycemia was proven to cause disruption of the blood-brain barrier (24). The permeability of structurally similar endoneurium may also be increased in diabetic patients, and the increase in serum CysC levels in DPN may be partly resulted from increase in endonurial fluid CysC concentration. Furthermore, we demonstrated that high serum CysC concentration was associated with DPN. In our study, DPN was assessed by nerve conduction velocity and amplitude of compound action potentials of peripheral nerves, which were characteristic electrophysiological traits of nerve fiber injury in DPN (2). Hence, elevated serum CysC concentration may indicate diabetic peripheral nerve injury and associated demyelination. In the CNS, CysC was mainly produced by neurons and microglia upon injury, and then released into the cerebralspinal fluid (25). Analogously, in peripheral neural diseases $\mathrm{Cys} C$ could be released from neurons and affected

Table 5 DPN risk in different CysC quartiles.

\begin{tabular}{|c|c|c|c|c|c|c|}
\hline & \multicolumn{6}{|c|}{ Confirmed DPN } \\
\hline & \multicolumn{2}{|l|}{ Total } & \multicolumn{2}{|l|}{ Male } & \multicolumn{2}{|l|}{ Female } \\
\hline & Odds ratio $(95 \% \mathrm{CI})$ & $P$ & Odds ratio $(95 \% \mathrm{Cl})$ & $P$ & Odds ratio $(95 \% \mathrm{Cl})$ & $P$ \\
\hline Quartile 1 & 1 & - & 1 & - & 1 & - \\
\hline Quartile 2 & $1.753(1.055-2.912)$ & 0.030 & $1.964(0.994-3.883)$ & NS & $1.541(0.714-3.326)$ & NS \\
\hline Quartile 3 & 2.463 (1.445-4.917) & 0.001 & $2.789(1.328-5.858)$ & 0.007 & 2.220 (1.017-4.848) & 0.045 \\
\hline Quartile 4 & $5.867(2.075-16.589)$ & 0.001 & 8.643 (1.15-64.953) & 0.036 & $5.250(1.505-18.312)$ & 0.009 \\
\hline
\end{tabular}



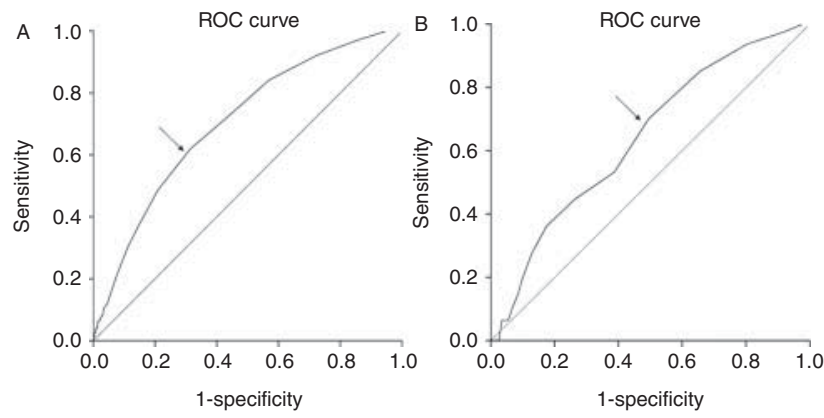

\section{Figure 1}

(A) ROC analysis of cystatin C (CysC) to indicate DPN for male patients. $A U C=0.704 ; 95 \% \mathrm{Cl}, 0.645-0.763$; identified CysC cutoff value $=1.25 \mathrm{mg} / \mathrm{l}$; Youden index $=0.305$; sensitivity: $61.8 \%$; specificity: $68.6 \%$. (B) ROC analysis of CysC to indicate DPN for female patients. $A \cup C=0.639 ; 95 \% \mathrm{Cl}, 0.559-0.719$; identified CysC cutoff value $=1.05 \mathrm{mg} / \mathrm{l}$; Youden index $=0.256$; sensitivity, $70.2 \%$; specificity, $50.43 \%$.

Schwann cells into endonurial fluid through the endoneurium.

There were several confounding factors in the association between DPN and serum CysC concetration. First of all, serum CysC was a more sensitive marker than $\mathrm{Cr}$ for estimating GFR in type 2 diabetic patients (26). An early population-based cohort study from Mayo Clinic revealed that staged severity of diabetic neuropathy was associated with diabetic nephropathy (3). Therefore, the relationship between DPN and serum CysC concentration may be attributed to the close association between DPN and diabetic nephropathy. However, although serum CysC concentration was closely related to GFR, and GFR was significantly lower in DPN patients, neither GFR nor Cr was an independently associated factor of DPN. And serum CysC was still associated with DPN after adjusting for renal function, which indicated that DPN was at least partly independently associated with serum CysC concentration. Moreover, cardiovascular diseases were also demonstrated to be associated with both DPN and serum CysC concentration $(2,9)$. The prospective study from the EURODIAB Prospective Complications Study Group identified that the incidence of neuropathy was associated with potentially modifiable cardiovascular risk factors, including a raised TG level and hypertension (2). In our study, although the prevalence of hypertension was significantly higher in DPN patients, hypertension was not an independently associated factor of DPN. And no difference in serum lipids concentrations was observed among three groups. There might be two explanations for this: Firstly, no studies proved that hypertension or dyslipidemia was independently associated with DPN. Secondly, treatment history including the antihypertensive and lipid-regulating therapies was not controlled in this cross-sectional study. On the other side, some potential confounders that may influence the serum CysC concentration and DPN, as suggested in previous research including age, sex, diabetes duration, HbA1c, GA, were excluded, and other neural diseases were also excluded at the time of DPN evaluation. Therefore, it can be concluded that the association between DPN and serum CysC concentration was at least partly independent of other variables. Moreover, we found that the cutoff point of serum CysC concentration to indicate DPN was higher in male patients. Although some studies revealed that serum CysC levels were generally not affected by extra-renal factors such as age and sex (27), a large-scale cross-sectional study in The Netherlands found that older age, male gender, etc. were independently associated with higher serum CysC levels after adjusting for $\mathrm{Cr}$ clearance (28). Therefore, the difference in cutoff points between male and female patients may be attributed to the association between serum CysC concentration and sex. As an indicator of renal dysfunction, particularly in diabetes, mircro-albuminuria had a significant association with DPN independent of other diabetic complications (5). However only male patients with persistent albuminuria and female patients with renal dysfunction had slightly higher DPN risk, and both male and female patients with high serum CysC concentration had a onefold higher risk of DPN,

Table 6 Odds ratio analysis of high serum cystatin C, persistent albuminuria, and renal dysfunction for the risk of confirmed DPN.

\begin{tabular}{|c|c|c|c|c|c|c|}
\hline & \multicolumn{6}{|c|}{ Confirmed DPN } \\
\hline & \multicolumn{2}{|l|}{ Total } & \multicolumn{2}{|l|}{ Male } & \multicolumn{2}{|l|}{ Female } \\
\hline & Odds ratio $(95 \% \mathrm{Cl})$ & $P$ value & Odds ratio $(95 \% \mathrm{Cl})$ & $P$ value & Odds ratio $(95 \% \mathrm{Cl})$ & $P$ value \\
\hline High cystatin C & - & - & $8.64(1.15-65.00)$ & 0.036 & $5.25(1.51-18.31)$ & 0.009 \\
\hline Persistent albuminuria & $1.187(0.441-3.195)$ & NS & $2.26(1.10-4.65)$ & 0.031 & $1.187(0.441-3.195)$ & NS \\
\hline Renal dysfunction & $1.795(1.075-2.996)$ & 0.029 & $2.00(0.84-4.80)$ & NS & $1.03(1.01-1.04)$ & 0.001 \\
\hline
\end{tabular}

www.eje-online.org 
indicating that high serum CysC concentration was a more important risk factor rather than persistent albuminuria and renal function. In general, the inter-assay coefficient of variation (CV) and intra-assay $\mathrm{CV}$ of CysC assay are 6.5 and $8.7 \%$ respectively.

There were some limitations in our study. First of all, only type 2 diabetic patients were enrolled for the screening of DPN, therefore the result of our study may not be applicable for other types of diabetes. Secondly, the ethnicity of the study population was relatively limited as they were mainly from local Shanghai. As a cross-sectional study, some other confounding factors of DPN, such as vitamin B12 deficiency and previous medical treatment for DPN, were not excluded.

In conclusion, we found that serum CysC levels increased significantly in type 2 diabetic patients with DPN, and high serum CysC level indicates significantly increased risk of DPN. Therefore, high serum CysC level may be a potential biomarker of DPN at least in type 2 diabetes. Further studies may reveal the immanent connection of CysC with the pathology of diabetic peripheral nerve injury and help to explore new treatment strategies for DPN.

\section{Declaration of interest}

The authors declare that there is no conflict of interest that could be perceived as prejudicing the impartiality of the research reported.

\section{Funding}

This study was supported by the National Natural Science Foundation of China (81270397 for F Liu, 81170759 for L Li).

\section{Author contribution statement}

Y Hu designed the study, researched data, and wrote, reviewed, and edited the manuscript. F Liu is the guarantor of this work, directed the research, and reviewed and edited the manuscript. J Shen conducted all the VPT value assessment, collected all the data, and took responsibility for the integrity of the data. $\mathrm{H}$ Zeng and $\mathrm{F}$ Lu conducted the neurological assessment. L Li, J Zhao, J Zhao, and $\mathrm{W}$ Jia reviewed the manuscript.

\section{References}

1 Icks A, Haastert B, Trautner C, Giani G, Glaeske G \& Hoffmann F. Incidence of lower-limb amputations in the diabetic compared to the non-diabetic population. findings from nationwide insurance data, Germany, 2005-2007. Expereimental and Clinical Endocrinology \& Diabetes 2009117 500-504. (doi:10.1055/s-0029-1225333)

2 Tesfaye S, Chaturvedi N, Eaton SE, Ward JD, Manes C, Ionescu-Tirgoviste C, Witte DR, Fuller JH \& EURODIAB Prospective Complications Study Group. Vascular risk factors and diabetic neuropathy. New England Journal of Medicine 2005352 341-350. (doi:10.1056/NEJMoa032782)
3 Dyck PJ, Kratz KM, Karnes JL, Litchy WJ, Klein R, Pach JM, Wilson DM, O'Brien PC, Melton LJ III \& Service FJ. The prevalence by staged severity of various types of diabetic neuropathy, retinopathy, and nephropathy in a population-based cohort: the Rochester Diabetic Neuropathy Study. Neurology 199343 817-824. (doi:10.1212/WNL.43.4.817)

4 Kärvestedt L, Mårtensson E, Grill V, Elofsson S, von Wendt G, Hamsten A \& Brismar K. Peripheral sensory neuropathy associates with micro- or macroangiopathy. Diabetes Care 200932 317-322. (doi:10.2337/dc08-1250)

5 Bell DS, Ketchum CH, Robinson CA, Wagenknecht LE \& Williams BT. Microalbuminuria Associated With Diabetic Neuropathy. Diabetes Care 199215 528-531. (doi:10.2337/diacare.15.4.528)

6 Tangri N, Stevens LA, Schmid CH, Zhang YL, Beck GJ, Greene T, Coresh J \& Levey AS. Changes in dietary protein intake has no effect on serum cystatin $\mathrm{C}$ levels independent of the glomerular filtration rate. Kidney International 201179 471-477. (doi:10.1038/ki.2010.431)

7 Dharnidharka VR, Kwon C \& Stevens G. Serum cystatin C is superior to serum creatinine as a marker of kidney function: a meta-analysis. American Journal of Kidney Diseases 200240 221-226. (doi:10.1053/ ajkd.2002.34487)

8 Shlipak MG, Sarnak MJ, Katz R, Fried LF, Seliger SL, Newman AB, Siscovick DS \& Stehman-Breen C. Cystatin C and the risk of death and cardiovascular events among elderly persons. New England Journal of Medicine 2005352 2049-2060. (doi:10.1056/NEJMoa043161)

9 Ix JH, Shlipak MG, Chertow GM \& Whooley MA. Association of cystatin $\mathrm{C}$ with mortality, cardiovascular events, and incident heart failure among persons with coronary heart disease: data from the Heart and Soul Study. Circulation 2007115 173-179. (doi:10.1161/CIRCULATIONAHA.106.644286)

10 Deng A, Irizarry MC, Nitsch RM, Growdon JH \& Rebeck GW. Elevation of cystatin C in susceptible neurons in Alzheimer's disease. American Journal of Pathology 2001159 1061-1068. (doi:10.1016/S00029440(10)61781-6)

11 Nakashima I, Fujinoki M, Fujihara K, Kawamura T, Nishimura T, Nakamura M \& Itoyama Y. Alteration of cystatin C in the cerebrospinal fluid of multiple sclerosis. Annals of Neurology 200762 197-200. (doi:10.1002/ana.20955)

12 Mi W, Pawlik M, Sastre M, Jung SS, Radvinsky DS, Klein AM, Sommer J, Schmidt SD, Nixon RA, Mathews PM et al. Cystatin C inhibits amyloid$\beta$ deposition in Alzheimer's disease mouse models. Nature Genetics 2007 39 1440-1442. (doi:10.1038/ng.2007.29)

13 Straface E, Matarrese P, Gambardella L, Vona R, Sgadari A, Silveri MC \& Malorni W. Oxidative imbalance and cathepsin D changes as peripheral blood biomarkers of Alzheimer disease: a pilot study. FEBS Letters 2005 579 2759-2766. (doi:10.1016/j.febslet.2005.03.094)

14 Yang Y, Liu S, Qin Z, Cui Y, Qin Y \& Bai S. Alteration of cystatin C levels in cerebrospinal fluid of patients with Guillain-Barré Syndrome by a proteomical approach. Molecular Biology Reports 200936 677-682. (doi:10.1007/s11033-008-9228-1)

15 Liu F, Shen J, Zhao J, Zeng H, Li L, Zhao J, Lu F, Bao Y \& Jia W. Cystatin C: a strong marker for lower limb ischemia in Chinese type 2 diabetic patients? PLoS One 20138 e66907. (doi:10.1371/journal.pone. 0066907)

16 He R, Shen J, Zhao J, Zeng H, Li L, Zhao J, Liu F \& Jia W. High cystatin C levels predict severe retinopathy in type 2 diabetes patients. European Journal of Epidemiology 201328 775-778. (doi:10.1007/ s10654-013-9839-2)

17 American Diabetes Association Standards of medical care in diabetes 2014. Diabetes Care 201437 (Suppl 1) S14-S80. (doi:10.2337/dc14-S014)

18 Martin CL, Albers JW, Pop-Busui R \& DCCT/EDIC Research Group. Neuropathy and related findings in the diabetes control and complications trial/epidemiology of diabetes interventions and complications study. Diabetes Care 201437 31-38. (doi:10.2337/dc13-2114)

19 Grubb A, Blirup-Jensen S, Lindström V, Schmidt C, Althaus H, Zegers I \& IFCC Working Group on Standardisation of Cystatin C (WG-SCC). First certified reference material for cystatin $\mathrm{C}$ in human serum 
ERM-DA471/IFCC. Clinical Chemistry and Laboratory Medicine 201048 1619-1621. (doi:10.1515/CCLM.2010.318)

20 Bril V \& Perkins BA. Validation of the Toronto Clinical Scoring System for diabetic polyneuropathy. Diabetes Care 200225 2048-2052. (doi:10.2337/diacare.25.11.2048)

21 Apelqvist J, Bakker K, van Houtum WH, Schaper NC \& International Working Group on the Diabetic Foot (IWGDF) Editorial Board. Practical guidelines on the management and prevention of the diabetic foot: based upon the International Consensus on the Diabetic Foot (2007) prepared by the international working group on the diabetic foot. Diabetes/Metabolism Research and Reviews 200824 (Suppl 1) S181-S187. (doi:10.1002/dmrr.848)

22 Shen J, Hu Y, Liu F, Zeng H, Li L, Zhao J, Zhao J, Zheng T, Lu H, Lu F et al. Vibration perception threshold for sight-threatening retinopathy screening in type 2 diabetic outpatients. Diabetes/Metabolism Research and Reviews 201329 525-531. (doi:10.1002/dmrr.2423)

23 Xiaofu T. Clinical eceltromyography study. Beijing: The Beijing University of Science and Technology China harmony Medical college unites the publishing house, 1995. pp 51-60.

24 Wunderlich MT, Ebert AD, Kratz T, Goertler M, Jost S \& Herrmann M. Early neurobehavioral outcome after stroke is related to release of neurobiochemical markers of brain damage. Stroke 199930 1190-1195. (doi:10.1161/01.STR.30.6.1190)

25 Deng A, Irizarry MC, Nitsch RM, Growdon JH \& Rebeck GW. Elevation of cystatin C in susceptible neurons in Alzheimer's disease. American Journal of Pathology 2001159 1061-1068. (doi:10.1016/S00029440(10)61781-6)

26 Mussap M, Dalla Vestra M, Fioretto P, Saller A, Varagnolo M, Nosadini R $\&$ Plebani $\mathrm{M}$. Cystatin $\mathrm{C}$ is a more sensitive marker than creatinine for the estimation of GFR in type 2 diabetic patients. Kidney International 200261 1453-1461. (doi:10.1046/j.1523-1755.2002. 00253.x)

27 Tangri N, Stevens LA, Schmid CH, Zhang YL, Beck GJ, Greene T, Coresh J \& Levey AS. Changes in dietary protein intake has no effect on serum cystatin C levels independent of the glomerular filtration rate. Kidney International 201179 471-477. (doi:10.1038/ ki.2010.431)

28 Knight EL, Verhave JC, Spiegelman D, Hillege HL, de Zeeuw D, Curhan GC \& de Jong PE. Factors influencing serum cystatin C levels other than renal function and the impact on renal function measurement. Kidney International 200465 1416-1421. (doi:10.1111/ j.1523-1755.2004.00517.x)

Received 12 May 2014

Revised 22 August 2014

Accepted 1 September 2014 